\title{
EFFECT OF DEXAMETHASONE INFILTRATION ON PERIODONTAL LIGAMENT AFTER EXPERIMENTAL MOLAR LUXATION IN RATS
}

\author{
Heba M. Hakam*
}

\begin{abstract}
Aim of the study: Dexamethasone is a popular corticosteroid in dental practice. Submucosal infiltration of dexamethasone proved to be effective to relief post-operative symptoms in different clinical and research application. This study was conducted to evaluate the effect of submucosal dexamethasone single injection on the healing of injured periodontal ligament fibers after molar luxation in rats via histological and gene expression studies.
\end{abstract}

Materials and methods:24 Wistar rats were selected for the study. Luxation injuries were performed for all first mandibular molars of both mandibular sides, right \& left. Immediately after luxation, the right sides of the mandibles received submucosal injections of dexamethasone sodium phosphate $(0.4 \mathrm{mg} / \mathrm{ml})$, while left sides were not dexamethasone injected. Follow up periods were 1,3 \& 7 days. Mandibular specimens were studied by routine histology, and quantitative gene expression analysis was done for fibroblast growth factor (FGF2), caspase-3 \& alkaline phosphatase (ALP).

Results: Histological features revealed superior results of the dexamethasone group with better regeneration and organization of fibers. Gene expression results revealed significant increase in the expression of both FGF2 and alkaline phosphatase (ALP) and a significant decrease in the expression of caspase- 3 in the study side when compared to the control side.

Conclusion: histologically dexamethasone enhanced the regeneration of torn periodontal ligament fibers with noticeable reduction in inflammation and rise in cellular proliferation. Gene expression reflected the possible benefit of dexamethasone single local injection in the management of periodontal ligament injuries.

KEYWORDS: dexamethasone, rat, luxation, periodontal ligament, alkaline phosphatase, FGF2, caspase-3.

\footnotetext{
* MD, MS, PhD, Associate Professor, Oral Biology Department, Faculty of dentistry, Cairo University
} 


\section{INTRODUCTION}

Corticosteroids in dentistry are usually used to alleviate post-operative symptoms in minor surgeries, endodontic periapical surgery ${ }^{2}$ and mucogingival surgery ${ }^{3}$. Topical corticosteroids are also used in dentistry for their immuno- suppressive and antiproliferative property ${ }^{4}$

Dexamethasone is one popular corticosteroid due to its market availability and reasonable cost. Clinical and experimental applications of dexamethasone reveal variable outcomes depending on its dosage, route as well as frequency of administration. ${ }^{4}$

Local injection of dexamethasone, in dental practice has been experimented for its efficacy in reduction of postoperative inflammatory symptoms since the middle of the last decade ${ }^{5,6,1}$. Studies on submucosal dexamethasone injection demonstrated its efficacy in reliving postoperative pain, swelling and trismus when it was compared to intra-muscular route. $^{7}$

In vitro experiments on periodontal ligament cells dealt with dexamethasone as an osteogenic inducer and as regeneration promoter in various researches. $^{8}$

The effect of dexamethasone is always modified according to dose amount and frequency variation that makes it an osteoporotic drug ${ }^{9}$ upon repeated and prolonged administration. On the other hand, it may be an osteogenic and healing promoter on lower doses with shorter duration. ${ }^{10,11}$

Although dexamethasone is widely used in dentistry, clinical and experimental, yet its introduction with periodontal problems is still limited. ${ }^{4}$

Experimental studies on tooth movement are applied in dental research to evaluate different tissue changes and understand physiologic events and periodontal adaptation accompanying such movements ${ }^{12}$. These researches are useful for periodontal, orthodontic and dental trauma clinical aspects. However, the traumatized supporting structure is not fulfilled till the present concerning survival under treatment and clinical follow up. The present research is designed to study the effect of local dexamethasone injection on periodontal ligament after molar luxation in rats.

\section{MATERIALS \& METHODS}

Animals: 24 Wistar rats were purchased for the study,120-150gm weight \&12-16 weeks age, from the animal house of Faculty of Medicine-Cairo University. The animals were maintained in an airconditioned animal house under controlled room temperature $25 \pm 2^{\circ} \mathrm{C}$ with $12 / 12 \mathrm{~h}$ light/dark cycle with free access to water and softened food. Rats were housed in standard polycarbonate cages as 3 animals per cage. General animal care was according to the guidelines of the institutional animal care and use committee of Cairo University.

Sample size was calculated using the ( $\mathrm{G}$ power software). We found that 8 rats for each duration was appropriate sample size for the study with total sample size 24 rats (1,3 and 7 days). The power is $80 \%$ and $\alpha$ error probability $=0.05$. the effect size $=1.52$ with each rat representing the control and the study groups.

\section{Experimental procedure:}

Animals were anesthetized by intraperitoneal injection of intraperitoneal injection of ketamine/ xylazine (ketamine 40-100 mg/kg IP/ xylazine 5-13 $\mathrm{mg} / \mathrm{kg}$ IP, Trittau, Germany) To induce periodontal injury each rat's first mandibular molar was gently luxated bucco-lingually as an incomplete extraction movement using a Mosquito hemostat. Luxation was considered done when the tooth is raised about $0.5 \mathrm{~mm}$ in its socket ${ }^{12}$.

Then luxation was performed on both mandibular sides taking the right side as experimental and the left as control. On the right mandibular sides supra-periosteal infiltration of $50 \mu 1(0.4 \mathrm{mg} / \mathrm{ml})$, of dexamethasone sodium phosphate (AMRIA pharmaceuticals, Egypt) into the buccal alveolar mucosa 
opposite to the mandibular $1^{\text {st }}$ molar ${ }^{13}$. Dexamethasone was diluted $1: 10$ with sterile saline to provide a maximum dose of $0.02 \mathrm{mg} /$ injection. Injection was performed using a micro-syringe and the solution slowly injected upon touching the bone surface. The exact procedures were performed on the left sides except for dexamethasone. Molars with broken or damaged roots were excluded from the study.

Animals were euthanized $1,3 \& 7$ days postoperatively, 8 animals/interval. The mandibles were sectioned each into two halves as the left is considered as control (untreated) and the right as experimental (treated). Then mandibles were prepared for histological examination and for quantitative analysis of gene expression of FGF, caspase 3 \& Alkaline phosphatase by real time polymerase chain reaction (PCR)

Histological examination: dissected mandibles were decalcified in $10 \%$ ethylene diamine tetraacetic acid (EDTA) for 3 weeks. Then dehydrated in alcohol, cleared in xylol and embedded in paraffin. Sections of $4-5 \mu$ thickness were mounted on regular glass slides and stained by Hematoxylin and Eosin for routine examination according to the conventional method.

\section{Quantitative analysis of gene expression of FGF} 2, Caspase 3 and ALP by real time PCR:

\section{Total RNA extraction}

Total RNA was extracted according to manufacturer instructions using SV Total RNA isolation system. (Promega, Madison, WI, USA). The RNA concentrations and purity were measured with an ultraviolet spectrophotometer.

\section{Complementary DNA (cDNA) synthesis}

The cDNA was synthesized from $1 \mu \mathrm{g}$ RNA using SuperScript III First-Strand Synthesis System as described in the manufacturer's protocol (\#K1621, Fermentas, Waltham, MA, USA).

\section{Real-time quantitative PCR (RT-PCR)}

RT-PCR amplification was done with Applied Biosystem with software version 3.1 (StepOne $^{\mathrm{TM}}$, USA). The reaction includes SYBR Green Master Mix and gene-specific primer pairs (Table 1). It was designed by Gene Runner Software (Hasting Software, Inc., Hasting, NY) from RNA sequences of the gene bank. Every set of primers exhibited $60^{\circ}$ annealing temperature. RT-PCR was done in $25-\mu 1$ reaction volume formed of 2 X SYBR Green PCR Master Mix, $900 \mathrm{nM}$ of every primer plus $2 \mu \mathrm{l}$ of complementary DNA. Amplification was applied under specific time and temperature conditions of several cycles of denaturation and annealing/ extension. $v 1 \cdot 7$ sequence detection software from PE Biosystems (Foster City, CA) was used to calculate the records of RT assays. Comparative $\mathrm{Ct}$ technique was used to determine the relative expression of mRNA gene. Data were standardized to beta actin which is the control housekeeping gene.

TABLE (1) Showing the primer sequence of the studied genes

\begin{tabular}{|l|l|}
\hline \multicolumn{2}{|c|}{ Primer sequence } \\
\hline \multirow{2}{*}{ FGF2 } & $\begin{array}{l}\text { F - 5'GCGGCTCTACTGCAAGA-3', } \\
\text { R -5'-CGTCCATCTTCCTTCATAGC-3', }\end{array}$ \\
\hline Caspase- 3 & $\begin{array}{l}\text { F: 5'-GTGGAACTGACGATGATATGGC-3' } \\
\text { R:5'-CGCAAAGTGACTGGATGAACC-3' }\end{array}$ \\
\hline $\begin{array}{l}\text { Alkaline } \\
\text { phosphatase }\end{array}$ & $\begin{array}{l}\text { F -5-CAGTGTCAGCCGTTAATTGAC-3 } \\
\text { R - 5-AGAGTAGGCTCCCGCCACG-3 }\end{array}$ \\
\hline \multirow{2}{*}{ Beta actin } & $\begin{array}{l}\text { F-5-GCGCTCGTCGTCGACAACGG-3 } \\
\text { R-5-GATGGCTACGTACATGGCTG-3 }\end{array}$ \\
\hline
\end{tabular}

\section{Statistical analysis}

Microstat7 for Windows statistical package was used for statistical analysis n this study. One-way ANOVA was used to evaluate effect of time on the selected parameters in each group. Paired " $\mathrm{t}$ " test was used to compare both groups in different intervals. Differences were considered statistically significant at $\mathrm{p} \leq 0.05^{14}$. 


\section{RESULTS}

\section{Histological results}

Longitudinal jaw sections revealed the area of the luxated molars with variable changes along the different follow up periods.

\section{One day post-operatively}

\section{Control untreated}

The periodontal fibers were torn around the roots with irregular pattern and diffuse hemorrhagic areas and evidence fibroblast degeneration with pyknotic nuclei. Aggregations of inflammatory cells concentrated around the traumatized area with widening of the interstitial spaces. Occasional osteoclasts were distributed along the pdl alveolar bone margin (fig.1).

\section{Dexamethasone treated}

The histological presentation of this group was like that of the control with apparent fewer hemorrhage spots despite the dilated capillaries that exhibited obvious fenestrations. Lesser inflammatory infiltration was also noticed with dexamethasone. Few pyknotic nuclei were also detected within fibroblasts (fig.1).

\section{3 days post-operatively}

\section{Control untreated}

Inflammatory infiltration was a characteristic sign of the current interval. The inflammatory cells were overlapping collagen fibers that exhibited discrete areas of hyalinization. The fibroblasts appeared with pyknotic nuclei in different locations around the roots. Sporadic osteoclasts on the alveolar bone surface were also detectable. (fig.2)

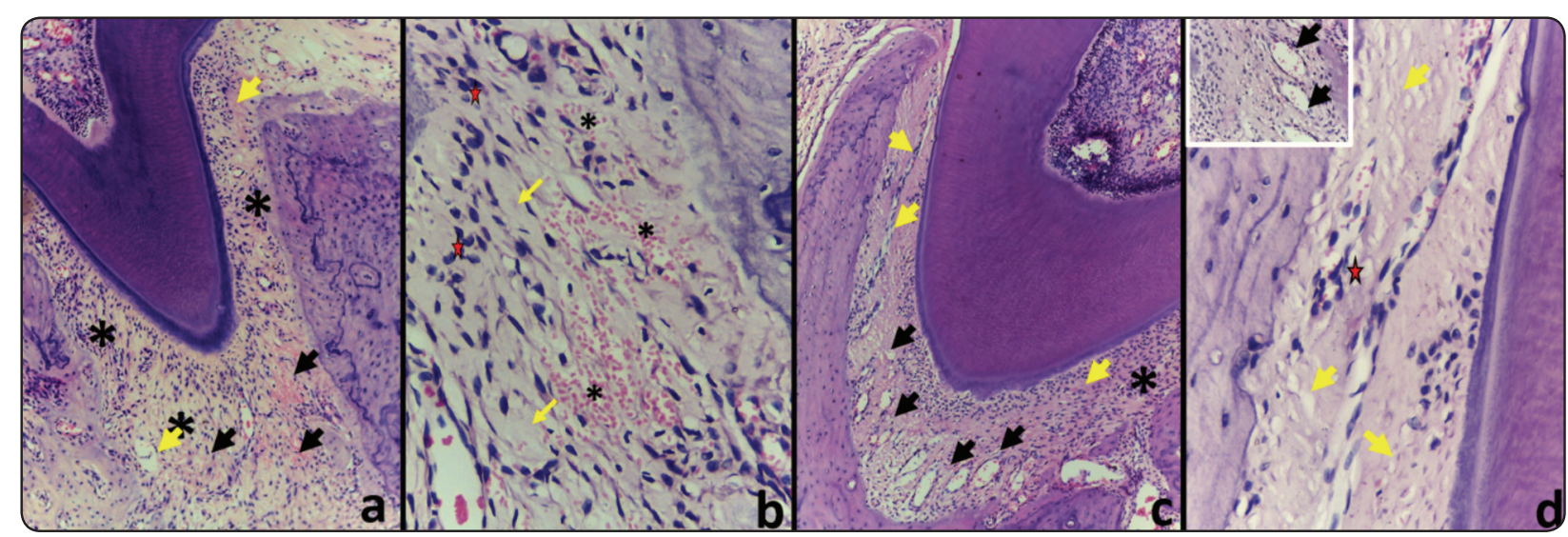

Fig. (1) Photomicrographs of the one-day interval showing

a) Control (untreated) group showing; inflammatory infiltration (asterisks), areas of hemorrhage (black arrows) \& torn collagen fibers (yellow arrows).

b) Higher magnification of control group showing; torn collagen fibers (yellow arrows), hemorrhage (asterisks) \& inflammatory cells (red stars).

c) Dexamethasone treated group showing; inflammatory infiltration (asterisks), dilated interstitial spaces with lesser congestion (black arrows) \& torn collagen fibers (yellow arrows).

d) Higher magnification of the Dexamethasone treated group showing; torn collagen fibers (yellow arrows), inflammatory cells (red stars) \& dilated interstitial space(inset). (H\&E orig. mag. a \& c, 100X, b \& d, 400X) 


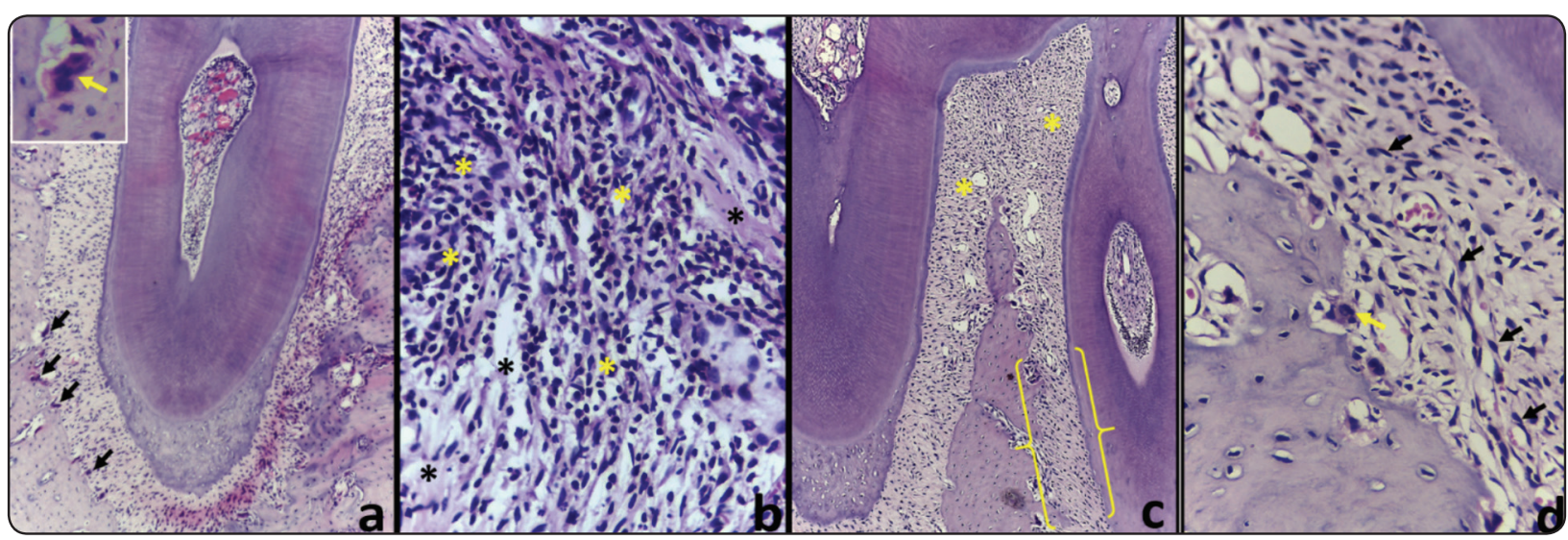

Fig. (2) Photomicrographs of the three-days interval showing

a) Control group showing; inflamed periodontal ligament around the root with osteoclasts on bone surface (black arrows), osteoclast (inset).

b) Higher magnification of control group showing; inflammatory infiltration (yellow asterisks) \& hyalinized collagen fibers (black asterisks).

c) Dexamethasone treated group showing; zones of proliferation and condensation (yellow asterisks) \& organized oblique group of fibers (yellow double brace)

d) Higher magnification of dexamethasone treated group showing; collagen fibers withspindle shaped fibroblasts (black arrows) \& osteoclast (yellow arrow). (H\&E orig. mag. a \& c, 100X, b \& d, 400X).

\section{Dexamethasone treated}

Apparently, the periodontal condition appeared characterized by increased proliferation of the fibroblasts with the characteristic spindle spindleshaped nuclei and increased density of collagen fibers as well. The fibers started to regain its attachment with both tooth and bone surfaces with some aligned as definite oblique fibers. A shaped that approached the well-known configuration. Fewer osteoclasts were evident than that in the untreated group (fig.2)

\section{7 days post-operatively}

\section{Control untreated}

Periodontal changes in the present interval included fiber regeneration with a bizarre pattern, as there was no definite identified pattern of fiber grouping seen. The ligament included multiple voids interrupting the continuity of collagen fibers around the root surface of the luxated molar. It was hard to identify interstitial spaces in the studied areas. (fig.3) some locations of the periodontal ligament appeared devoid of the nuclei of fibroblasts while other nuclei appeared pyknotic. Massive alveolar bone resorption was also a feature of the current group with evidence of osteoclasts. Multinucleated giant cells were detected within the periodontal ligament as well. (fig.3)

\section{Dexamethasone treated}

Periodontal healing in the current group showed more improvement when compared to the preceding group. More organized pattern of periodontal fibers with aligned fibroblasts with spindle nuclei. Pyknotic nuclei were less evident that the control group. The ligament appeared more intact than the untreated group except few locations where the fibers exhibited an irregular pattern. The adjacent alveolar bone exhibited a healthier appearance with multiple resting lines denoting its activity and osteoclasts were scarcely seen (fig.3) 


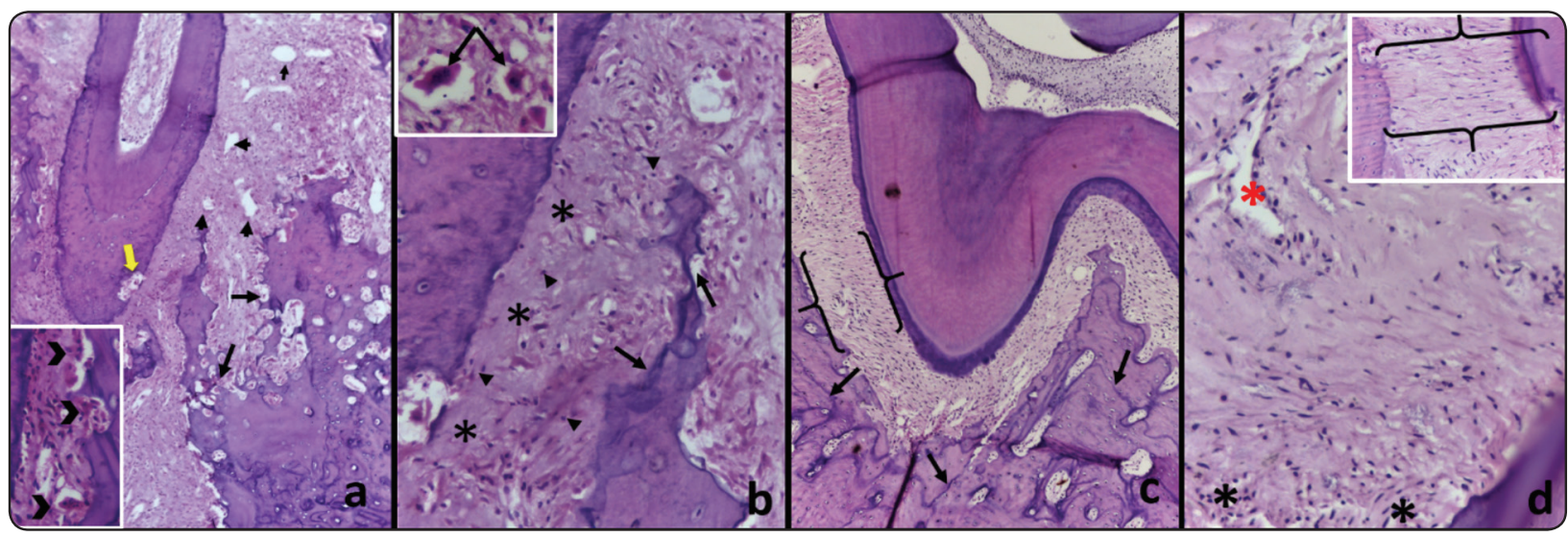

Fig. (3) Photomicrographs of the seven-days interval showing;

a) Control group showing; multiple voids in the periodontal ligament (short black arrows), resorption of the alveolar bone (long black arrows), root resorption (yellow arrow) \& osteoclasts (arrow heads-inset).

b) Higher magnification of control group showing; alveolar bone resorption (arrows), homogenized areas of periodontal ligament without cellular existence (asterisks), nuclear pyknosis (arrow heads) and osteoclast (inset)

c) Dexamethasone treated group showing; intact periodontal ligament with fewer voids with defined oblique group (double brace), resting bone lines (arrows).

d) Higher magnification of dexamethasone treated group showing; cellular proliferation (black asterisks) fewer discontinuity (red asterisk) \& organized horizontal fiber group (inset) (H\&E orig. mag. a \& c, 100X, b \& d, 400X).

Gene expression results: Comparisons between the study groups \& effect of time within the same group

FGF2: Comparing the two groups revealed that the highest mean value was recorded in treated group at 3 days, while the least value was recorded in untreated group at 1 day. The difference between groups was statistically significant (table 2). Within the same group, treated or untreated, the difference was not statistically significant (untreated: $\mathrm{p}=0.362$ ). (treated: $\mathrm{p}=0.083$ )

In both groups there was no significant between three intervals

The study group showed statistically significantly higher values than control group in all intervals. (table 2, fig. 4)

Caspase-3: Comparing the two groups revealed that the highest mean value was recorded in untreated group at 1 days, while the least value was recorded in treated group at 7 days. The difference between groups was statistically significant (table 3 ). Within the treated group, the highest mean value was recorded at day1, while the least value was recorded at day7 with no statistically significant difference.

In untreated group there was insignificant decrease after 3 days $(\mathrm{p}=0.033)$. There was no statistically significant difference between the three intervals $(\mathrm{p}=0.582)$. The treated group in general showed statistically significantly lower values than control group in all intervals (table 3 , fig. 5)

ALP: Comparing the two groups confirmed that the highest mean value was recorded in treated group at 7 days, while the least value was recorded in control. Within the untreated group, the highest mean value was recorded at 3 days, while the least value was recorded at day1. The difference by time was not statistically significant $(\mathrm{p}=0.868)$. Within the treated group, the mean value gradually increased by time, with no statistically significant difference $(p=0.211)$. In both groups there was no significant between three intervals. The study group showed statistically significantly higher values than control group in all intervals table (4, fig 6) 
TABLE (2): One-Way ANOVA evaluating effect of time on FGF in both groups and independent student " $t$ " test comparing both groups in each interval.

\begin{tabular}{|c|c|c|c|c|c|c|}
\hline \multirow{2}{*}{ FGF } & \multicolumn{2}{|c|}{ Control (untreated) } & \multicolumn{2}{|c|}{ Study(treated) } & \multirow{2}{*}{ "t $"$} & \multirow{2}{*}{ Probability } \\
\hline & Mean & St Dev & Mean & St Dev & & \\
\hline Day 1 & 2.04 & 0.15 & 5.43 & 0.25 & 20.019 & $0.0000 *$ \\
\hline Day 3 & 2.30 & 0.36 & 6.91 & 1.07 & 7.076 & $0.001 *$ \\
\hline Day 7 & 2.33 & 0.21 & 6.47 & 0.35 & 17.536 & $0.0000 *$ \\
\hline$F$ ratio & \multicolumn{2}{|c|}{1.211} & \multicolumn{2}{|c|}{3.885} & & \\
\hline Probability & \multicolumn{2}{|c|}{$0.362 \mathrm{NS}$} & \multicolumn{2}{|c|}{$0.083 \mathrm{NS}$} & & \\
\hline LSD & \multicolumn{2}{|c|}{0.511} & \multicolumn{2}{|c|}{1.709} & & \\
\hline
\end{tabular}

*= Significant $\quad$ NS $=$ Not significant

TABLE (3): One-Way ANOVA evaluating effect of time on Caspase 3 in both groups and independent student " $\mathrm{t}$ " test comparing both groups in each interval.

\begin{tabular}{|c|c|c|c|c|c|c|}
\hline \multirow{2}{*}{ Caspase3 } & \multicolumn{2}{|c|}{ Control(untreated) } & \multicolumn{2}{|c|}{ Study(treated) } & \multirow{2}{*}{ "t" } & \multirow{2}{*}{ Probability } \\
\hline & Mean & St Dev & Mean & St Dev & & \\
\hline Day 1 & 4.50 & 0.89 & 1.74 & 0.31 & 5.085 & $0.004 *$ \\
\hline Day 3 & 3.34 & 0.29 & 1.53 & 0.15 & 9.429 & $0.0000^{*}$ \\
\hline Day 7 & 2.93 & 0.25 & 1.50 & 0.36 & 5.646 & $0.002 *$ \\
\hline F ratio & \multicolumn{2}{|c|}{6.327} & \multicolumn{2}{|c|}{0.592} & & \\
\hline Probability & \multicolumn{2}{|c|}{$0.033^{*}$} & \multicolumn{2}{|c|}{$0.582 \mathrm{NS}$} & & \\
\hline LSD & \multicolumn{2}{|c|}{1.119} & \multicolumn{2}{|c|}{0.576} & & \\
\hline
\end{tabular}

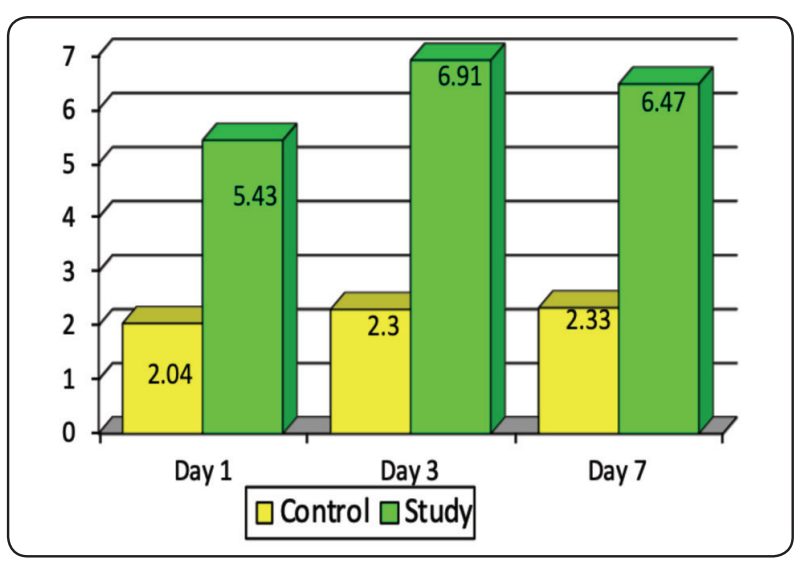

Fig. (4) Mean values of FGF2 in both groups during follow up intervals. Control refers to the untreated \& study refers to the dexamethasone treated.

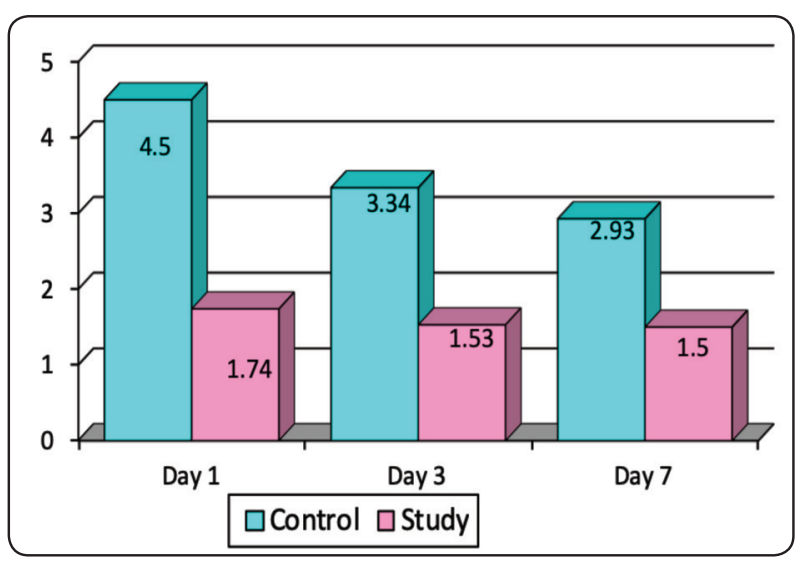

Fig. (5) Mean values of Caspase 3 in both groups during follow up intervals. Control refers to the untreated \& study refers to the dexamethasone treated. 
TABLE (4) One-Way ANOVA evaluating effect of time on Alkaline Phosphatase in both groups and independent student " $t$ " test comparing both groups in each interval.

\begin{tabular}{|c|c|c|c|c|c|c|}
\hline \multirow{2}{*}{$\begin{array}{c}\text { Alkaline } \\
\text { Phosphatase }\end{array}$} & \multicolumn{2}{|c|}{ Control(untreated) } & \multicolumn{2}{|c|}{ Study(treated) } & \multirow{2}{*}{ "t" } & \multirow{2}{*}{ Probability } \\
\hline & Mean & St Dev & Mean & St Dev & & \\
\hline Day 1 & 1.37 & 0.15 & 4.33 & 0.15 & 23.786 & $0.0000^{*}$ \\
\hline Day 3 & 1.48 & 0.39 & 4.53 & 0.38 & 9.679 & $0.0003 *$ \\
\hline Day 7 & 1.43 & 0.15 & 4.78 & 0.23 & 21.292 & $0.0000 *$ \\
\hline F ratio & \multicolumn{2}{|c|}{0.145} & \multicolumn{2}{|c|}{2.042} & & \\
\hline Probability & \multicolumn{2}{|c|}{$0.868 \mathrm{NS}$} & \multicolumn{2}{|c|}{$0.211 \mathrm{NS}$} & & \\
\hline LSD & \multicolumn{2}{|c|}{0.518} & \multicolumn{2}{|c|}{0.538} & & \\
\hline
\end{tabular}

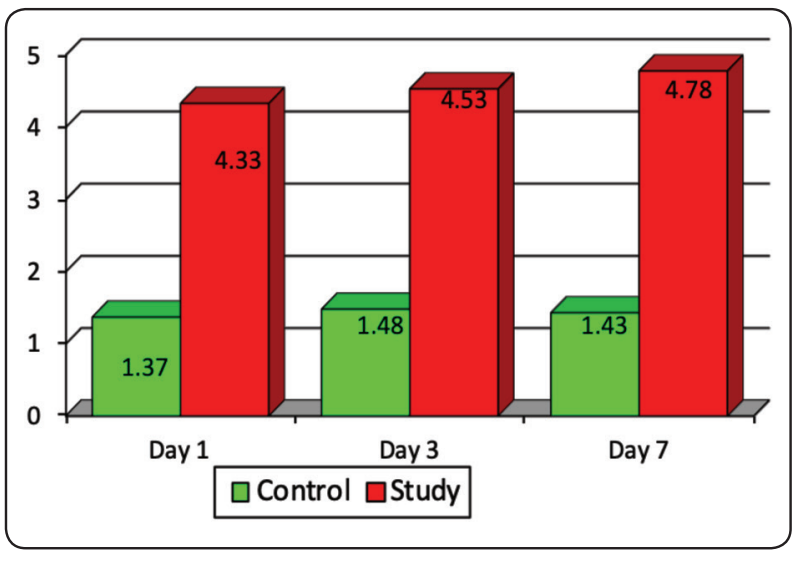

Fig. (6) Mean values of Alkaline Phosphatase in both groups during follow up intervals. Control refers to the untreated \& study refers to the dexamethasone treated.

\section{DISCUSSION}

The present study was based on multiple trials dealing with the use of local injection of dexamethasone in dentistry as an anti-inflammatory and an anti-edematous agent $1,7,3$.

Experimental work considers rat successful animal model is for being easily manipulated and a for obtaining similar tissues to the human body ${ }^{15}$ and different researches on dexamethasone effect used rats as the model of choice. ${ }^{2,16}$

The use of dexamethasone periodontal tissue was studied in different in vitro experiments ${ }^{17-18}$ and in vivo available research work dealt with the drug action as repeated injectable doses or as a topical application. ${ }^{4,19,20}$

Tooth luxation can be induced by trauma, infection, periodontitis, malocclusion or undue orthodontic force application. If the practitioner can overcome the hazards of tooth luxation by a simple technique that would be of a great value for the patient which developed the idea of the present research.

In the ongoing research mandibular first molar luxation was performed on both sides of the rat's mandible to induce periodontal ligament trauma. Submucosal dexamethasone was injected on the buccal alveolar mucosa on the right side opposite to the luxated tooth while the left side received no injection. Animals were euthanized at 1,3 and 7 days after procedure. The mandibles were dissected, and the area of interest was prepared for histological examination and for gene expression of fibroblast growth factor (FGF), caspase-3 and alkaline phosphatase activity.

The histological results of the current research revealed in general better reaction to dexamethasone injection over the follow up intervals. In the earlier follow up detection of hemorrhage and inflammation was seen in both treated and untreated groups with lesser capillary congestion and apparently less 
inflammatory infiltration I the dexamethasone treated group which coincides with the drug effect in different research work ${ }^{3,21-23}$. The detected shape of torn fibers and the hemorrhagic areas was also described by Nagayama et al, $2012^{15}$.

The current results are also in agreement with Harshita et al, $2018^{4}$ who explained that corticosteroids can induce the production of lipocortin, a polypeptide group, that block the action of vasodilators such as histamine and bradykinin which explains the decreased capillary congestion among the dexamethasone group.

Upon longer follow after three days the inflammation subsided in the treated group with dexamethasone better than that of the untreated group and this period was characterized by increased cellular proliferation and beginning of formation of new fibers which demonstrated the ability of dexamethasone to induce cellular proliferation. That coincided with other investigators who revealed the ability of dexamethasone to stimulate proliferation and regeneration of fibroblasts \& osteoblasts in culture ${ }^{24,8}$. the noticed decrease in inflammatory infiltration in this interval (three days) is in accordance with Hakim et al, $2005^{25}$ who detected a significant decrease in inflammatory markers at a similar interval, three days, when dexamethasone was injected into muscles after muscular injury.

Local injection of a single dose of dexamethasone after muscle injury was also helpful in restoring the contractile tension of the muscle after three days of injection which is further supported by the current results in the three days interval ${ }^{25}$.

Regenerative effect of dexamethasone also extended to nerve tissue as I.M. drug injection helped in the regeneration of crushed sciatic nerve in rat. The drug increased nerve function and controlled Walarian degeneration and enhanced the regeneration of myelinated nerve fibers ${ }^{26}$

Moreover, Hakki et al, $2009{ }^{27}$ suggested that dexamethasone maybe of advantage in periodontal regeneration as it inhibited the expression of matrix metalloproteinases MMP-1, 3 \& 9 which inhibited degeneration of periodontal ligament cells in culture. which further coincides with the herein findings.

Furthermore, Kirakozova et al, $2009^{28}$ studied the effect of intra-canal corticosteroids on periodontal healing in avulsed teeth in dogs. they concluded that canals filled with corticosteroids showed superior healing criteria when compared with gatta percha filled canals which supports the ongoing study findings.

On the other hand, the current findings were not in agreement with other investigators whose findings revealed that the administration of dexamethasone may inhibit soft tissue healing ${ }^{16}$ or delay extraction socket healing ${ }^{29}$. other researchers demonstrated that dexamethasone suppressed osteoblasts both in vivo and in vitro ${ }^{9,10}$.

However, on reviewing the mentioned researches it was evident that they depended on repeated or prolonged administration of the drug which explains the different outcome in the present work that depended on single dose application. Harshita et al, $2018{ }^{4}$ confirmed that the effect of corticosteroids on any tissue is usually a dose and concentration dependent effect which supports the mentioned assumption for the current work.

Reviewing the next follow up period, 7 days, noticeable changes were detected in both periodontal ligament and alveolar bone histology. healing of the periodontal ligament area was nearly established and approached its normal configuration which further supports the results in previous research work ${ }^{30}$. In addition, the ongoing results are in accordance with Kirakozova et al, 2018 who revealed that dexamethasone treatment improved periodontal healing of replanted teeth in dogs. They suggested that corticosteroids in general could be effective in stimulation of periodontal ligament recovery ${ }^{28}$. Moreover, the organized spindle shape of fibroblasts demonstrated in the current interval of the treated group was also recorded by other researchers' in vitro 
studies $^{27,31}$. However, the herein results of the treated group were not in accordance with Choi et al, as they reported that dexamethasone treatment induced the production of DKK-1 gene by periodontal ligament stem cells in vitro which in turn inhibited the growth of human periodontal ligament stem cells ${ }^{17}$.

Massive alveolar bone resorption was detected in the control, untreated, group while in the dexamethasone-treated group bone activity was normal represented by resting and reversal lines mapping the bone surface. moreover, the osteoclasts were more detected in the untreated group. This demonstrates the ability of the drug to arrest bone resorption that may result from traumatic tooth movement which supported previous research work ${ }^{11}$ that revealed that dexamethasone inhibited bone resorption during the early stage of orthodontic movement in rat.

Nuclear pyknosis was also shown in different locations of the periodontal ligament of the control group a finding that coincides with other investigators ${ }^{15}$.

Gene expression results supported the histological findings as well. The values of statistical analysis revealed statistically significant increase in the dexamethasone treated groups over the untreated groups regarding FGF2 and ALP expression means in general comparison between the two groups. However, within each group there was no statistically significant difference upon comparing the different follow up intervals. Additionally, the expression of Caspase 3 was noticed to be lower in the dexamethasone treated group that the untreated group with a statistically significant difference. Meanwhile, between each group intervals there was no statistical difference.

These results coincided with previous literature as periodontal ligament was proved to express FGF2 during healing of periodontal defects ${ }^{32}$. Furthermore, it was recorded by Hakki et al, $2009^{27}$ that the addition of dexamethasone to FGF2 to cultured periodontal ligament cells would enhance the periodontal regeneration. While the sole addition of dexamethasone induced biomineralization and osteogenic differentiation ${ }^{31}$.An observation that also explains the elevated means of alkaline phosphatase expression in the current study. Dexamethasone was demonstrated to improve periodontal healing in research work which is confirmed by the elevated level of FGF2 in the present study. Dexamethasone also resulted in increased levels of ALP in the treated group samples which was also demonstrated in previous research work ${ }^{33}$. The elevated level of ALP may be one of the factors that prevented the massive bone resorption detected in the untreated group. On the other hand, dexamethasone was found to lessen the ALP activity in female rats after repeated, twice weekly, injection ${ }^{10}$, which is not in accordance with the present findings.

The levels of caspase 3 were significantly lower among the treated group coinciding with other investigators ${ }^{34}$. However, other researches demonstrated that dexamethasone induced apoptosis in human periodontal ligament stem cells ${ }^{34}$.

In summery the present study suggests that a single local injection of dexamethasone can have a regenerative effect on the damaged periodontal ligament which can be of a therapeutic value in cases with traumatized periodontal ligament in luxated or avulsed teeth. This was based on the histological findings that were further confirmed by the PCR results that coincided with most of the reviewed research work results. The difference in the drug effect can vary according to its frequency, dosage and route of administration. Further investigation is needed with varying doses and longer follow up intervals.

\section{REFERENCES}

1. Warraich R, Faisal M, Rana M, Shaheen A. Evaluation of postoperative discomfort following third molar surgery using submucosal dexamethasone e a randomized observer blind prospective study. Oral Surgery, Oral Med Oral Pathol Oral Radiol. 2013;116(1):16-22. doi:10.1016/j. oooo.2012.12.007

2. Yavari HR, Jafari F, Jamloo H. The Effect of Submucosal Injection of Corticosteroids on Pain Perception and 
Quality of Life after Root Canal Treatment of Teeth with Irreversible Pulpitis : A Randomized Clinical Trial. J Endod. 2019;(14):1-7. doi:10.1016/j.joen.2019.01.005

3. Zardo LN, André F, Pilatti GL. Use of etoricoxib and dexamethasone for postoperative pain prevention and control in mucogingival surgery - A randomized parallel doubleblind clinical trial. 2013;12(4).

4. Harshita N, Kamath DG. Corticosteroids - Assets and Liabilities on Periodontium. 2018;11(8).

5. Majid OW, Mahmood WK. Effect of submucosal and intramuscular dexamethasone on postoperative sequelae after third molar surgery: Br J Oral Maxillofac Surg. 2011;49(8):647-652. doi:10.1016/j.bjoms.2010.09.021

6. Klongnoi B, Kaewpradub P, Boonsiriseth K. Effect of single dose preoperative intramuscular dexamethasone injection on lower impacted third molar surgery. Int J Oral Maxillofac Surg. 2012;41(3):376-379. doi:10.1016/j. ijom.2011.12.014

7. Troiano G, Laino L, Cicciù M, et al. Comparison of Two Routes of Administration of Dexamethasone to Reduce the Postoperative Sequelae After Third Molar Surgery: A Systematic Review and Meta-Analysis. 2018:181-188. doi:10.2174/1874210601812010181

8. Vito A Di, Giudice A, Chiarella E, Malara N, Bennardo F, Fortunato L. In Vitro Long-Term Expansion and High Osteogenic Potential of Periodontal Ligament Stem Cells : More Than a Mirage. 2019;28(1):129-139. doi: $10.1177 / 0963689718807680$

9. Liu J, Mattheos N, Su C, et al. The effects of icariin on wound healing of extraction sites with administration of zoledronic and dexamethasone : A rat model study. 2018; (November 2017):198-205. doi:10.1111/jop.12659

10. Pan J, Wu L, Cai J, Wu L, Liang M. Dexamethasone suppresses osteogenesis of osteoblast via the PI3K / Akt signaling pathway in vitro and in vivo. J Recept Signal Transduct. 2019;39(1):80-86. doi:10.1080/10799893.2019.1625061

11. Amorim L, Knop H, Shintcovsk RL, Retamoso LB, Ribeiro JS, Tanaka OM. Non - steroidal and steroidal anti -infl ammatory use in the context of orthodontic movement. 2012;34:531-535. doi:10.1093/ejo/cjq173

12. Miyashin M, Kato J, Experimental TY. Experimental luxation injuries in immature rat teeth. 1990:121-128.

13. Nobuhara WK, Carnes DL, Gilles JA. Anti-Inflammatory Effects of Dexamethasone on Periapical Tissues following Endodontic Overinstrumentation IrE r 11 Ift. 1993;19(10):501-507.
14. Chan YH. Biostatistics 102 : Quantitative Data - Parametric. 2003;44(8):391-396

15. Nagayama T, Seiryu M. Increase of CGRP-Containing Nerve Fibers in the Rat Periodontal Ligament After Luxation. 2012:391-397. doi:10.1007/s10571-011-9767-1

16. Shetty BS, Udupa SL, Udupa AL. Effect of Centella asiatica L ( Umbelliferae ) on Normal and Dexamethasone-Suppressed Wound Healing in Wistar Albino Rats. 2006;5(3):137-143. doi:10.1177/1534734606291313

17. Choi S, Park EK, Kwack MH, Sung YK. Effects of dexamethasone, a synthetic glucocorticoid, on human periodontal ligament stem cells. 2015:991-995. doi:10.1007/ s00210-015-1151-0

18. Kim B, Kim M, Park Y, Ko Y, Park J. Short-term application of dexamethasone on stem cells derived from human gingiva reduces the expression of RUNX2 and b -catenin. 2017. doi:10.1177/0300060517701035

19. Mergoni G, Vescovi P, Passerini P, et al. Effects of zoledronic acid and dexamethasone on early phases of socket healing after tooth extraction in rats : A preliminary macroscopic and microscopic quantitative study. 2019;24(3). doi: $10.4317 /$ medoral. 22883

20. Keum K, Kwon O, Spa LS, et al. Effect of Dexamethasone on Root Resorption After Delayed Replantation of Rat Tooth. 2003.

21. Lim D, Omfs M, Ngeow WC. SC. J Oral Maxillofac Surg. 2017. doi:10.1016/j.joms.2017.05.033

22. Lim D, Omfs M, Ngeow WC. DENTOALVEOLAR SURGERY A Comparative Study on the Efficacy of Submucosal Injection of Dexamethasone Versus Methylprednisolone in Reducing Postoperative Sequelae After Third Molar Surgery. J Oral Maxillofac Surg. 2017;75(11):22782286. doi:10.1016/j.joms.2017.05.033

23. Arakeri G, Rai KK, Shivakumar HR, Jayade B. A Randomized Clinical Trial to Compare the Efficacy of Submucosal Aprotinin Injection and Intravenous Dexamethasone in Reducing Pain and Swelling After Third Molar Surgery : A Prospective Study. 2013;12(1):73-79. doi:10.1007/ s12663-012-0364-8

24. Roozegar MA, Mohammadi TM, Havasian MR, Hashemian A, Amraei M, Hoshmand B. In vitro Osteogenic impulse effect of Dexamethasone on periodontal ligament stem cells. 2015;11(2).

25. Hakim M, Hage W, Lovering RM, Moorman CT, Curl LA, Deyne PG De. Dexamethasone and Recovery of Contractile Tension after. 2005;(439):235-242. doi:10.1097/01. blo.0000177716.70404.f9 
26. Feng X, Yuan W. Dexamethasone Enhanced Functional Recovery after Sciatic Nerve Crush Injury in Rats. 2015;2015. doi:10.1155/2015/627923

27. Ss H, Ee H, Rm N. Regulation of matrix metalloproteinases and tissue inhibitors of matrix metalloproteinases by basic fibroblast growth factor and dexamethasone in periodontal ligament cells. 2009:794-802 doi:10.1111/j.16000765.2008.01192.x

28. Kirakozova A, Teixeira FB, Curran AE, et al. Effect of Intracanal Corticosteroids on Healing of Replanted Dog Teeth after Extended Dry Times. J Endod. 2009;35(5):663667. doi:10.1016/j.joen.2009.01.009

29. Sonis ST, Watkins BA, Lyng GD, Lerman MA, Anderson $\mathrm{KC}$. Bony changes in the jaws of rats treated with zoledronic acid and dexamethasone before dental extractions mimic bisphosphonate-related osteonecrosis in cancer patients. Oral Oncol. 2009;45(2):164-172. doi:10.1016/j. oraloncology.2008.04.013
30. Uzun T, Toptas O, Saylan A. Evaluation and Comparison of the Effects of Artesunate, Dexamethasone, and Tacrolimus on Sciatic Nerve Regeneration. J Oral Maxillofac Surg. 2018; 77(5):1092.e1-1092.e12. doi:10.1016/j.joms.2018.12.019

31. Qin X, Li Q, Chen W, Bai Y, Baban B, Mao J. The circadian expression of osteogenic factors in periodontal tissue loading mechanical force : new concepts of the personalized orthodontic care. 2019:13-20.

32. Ren J, Chew J, Jiunn S, et al. Acta Biomaterialia Mesenchymal stem cell exosomes enhance periodontal ligament cell functions and promote periodontal regeneration. Acta Biomater. 2019;89:252-264. doi:10.1016/j.actbio.2019.03.021

33. Park JUNB. Effects of the combination of dexamethasone and fibroblast growth factor - 2 on differentiation of osteoprecursor cells. 2014:659-662. doi:10.3892/mmr.2013.1811

34. Kim S, Kim Y, Park J, Lee J, Suh J. The effects of dexamethasone on the apoptosis and osteogenic differentiation of human periodontal ligament cells. 2013:168-176. 